Communications in Physics, Vol. 28, No. 4 (2018), pp. 337-349

DOI:10.15625/0868-3166/28/4/12930

\title{
COMBINED SENSITIVITY OF T2K-II AND NO $v$ A EXPERIMENTS TO CP VIOLATION IN LEPTON SECTOR
}

\author{
TRAN VAN NGOC ${ }^{1}$, CAO VAN SON ${ }^{2}$, AND NGUYEN THI HONG VAN ${ }^{1,3, \dagger}$ \\ ${ }^{1}$ Institute For Interdisciplinary Research in Science and Education (IFIRSE) \\ Ghenh Rang, Quy Nhon, Vietnam \\ ${ }^{2}$ High Energy Accelerator Research Organization (KEK) \\ Tsukuba, Ibaraki, Japan \\ ${ }^{3}$ Institute of Physics, Vietnam Academy of Science and Technology \\ 10 Dao Tan, Ba Dinh, Hanoi, Vietnam \\ ${ }^{\dagger} E$-mail: nhvan@iop.vast.ac.vn \\ Received 04 August 2018 \\ Accepted for publication 21 September 2018 \\ Published 15 December 2018
}

\begin{abstract}
In this paper, we study the combined sensitivity of T2K-II and NOvA, the world leading long-baseline neutrino oscillation experiments, to CP violation in lepton sector. By operating until the year 2026, T2K-II is expected to collect a total exposure of $20 \times 10^{21}$ protons-on-target. Meanwhile, NOVA experiment has a plan to extend its run up to 2024 for accumulating totally an amount of $72 \times 10^{20}$ protons-on-target. By combining analyses of T2K-II and NOVA with an ultimate constraint on $\theta_{13}$ from reactor, the sensitivity to $C P$ violation is expected to exceed $4 \sigma$ C.L. It is also pointed out that by reducing the systematic uncertainties of both T2K-II and NOVA to a level of $2 \%$, the sensitivity to CP violation will significantly increase with a discovery possibility at $5 \sigma$ C.L. significance for a particular range of true $\delta_{C P}$.
\end{abstract}

Keywords: CP violation, neutrinos, T2K experiment, NOvA experiment.

Classification numbers: $14.60 . \mathrm{Pq}, 14.60 . \mathrm{Pq}, 25.30 . \mathrm{Pt}$.

\section{INTRODUCTION}

The idea of neutrino oscillation, in which one type of neutrino can change into another, was raised for the first time by B. Pontecorvo in 1957 as neutrino-antineutrino transition [1]. It can explain the solar neutrino anomaly [2,3] and the atmospheric neutrino anomaly [4]. The neutrino flavor oscillation was introduced later in 1962 by Z. Maki, M. Nakagawa and S. Sakata [5]. The neutrino oscillation phenomenon was observed by Super-Kamiokande experiment [6],

(C)2018 Vietnam Academy of Science and Technology 
SNO experiment [7] and later conclusively confirmed by number of neutrino experiments with different detection techniques at different energy ranges and different baselines. Discovery of neutrino oscillation which indicates that neutrinos have mass and mixing among states, matters a lot since this is the only experimental evidence for the incompleteness of the Standard Model of fundamental particles.

Except for some anomalies, the up-to-date (anti-)neutrino data from various experiments can be well described by a $3 \times 3$ unitary mixing matrix, so-called PMNS matrix. This unitary matrix, as discussed more details in the next section, are parameterized by three mixing angles $\left(\theta_{12}\right.$, $\theta_{13}$ and $\left.\theta_{23}\right)$ and one single Dirac phase $\delta_{\mathrm{CP}}{ }^{1}$ which represents CP violation in the lepton sector. The three mixing angles are determined to be non-zero [8] and this allows neutrino experiments to make measurement on the $\mathrm{CP}$ violation in the lepton sector, which is one of the most central objective at present and in near future of neutrino physics. Besides these four parameters, the oscillation probabilities depend on the mass-squared differences among the mass eigenstates, neutrino energy and distance travelled. At the current landscape of neutrino oscillation physics, two scales of mass-squared differences are determined. However their mass ordering is still unknown and also one of the most important questions need to be addressed in the future.

T2K and NOvA are two among the world leading neutrino experiments in searching the $\mathrm{CP}$ violation in the lepton sector. The combined sensitivity of these two experiments was performed and showed that this sensitivity can be up to $2 \sigma$ or higher if the true value $\delta_{C P}$ is about $-\pi / 2$ [9]. We are revising this analysis with three main updates including (i) possible $\mathrm{T} 2 \mathrm{~K}$ run extension up to 2026, so-called T2K-II and NOvA run extension up to 2024, (ii) improvement in selection performance and systematic uncertainties in both experiments and (iii) the ultimate precision on mixing angle $\theta_{13}$ can be achieved by the reactor measurements. The first twos are crucial since the measurement is dominated by the statistical errors. The third one is needed to break down the $\delta_{C P}-\theta_{13}$ degeneracy with accelerator-based long baseline experiments.These combined critical factors enhance capability to search $\mathrm{CP}$ violation to unprecedented level of sensitivity.

The paper is organised as follows: In section II, the PMNS formalism of neutrino oscillation is introduced with an intense focus on how CP violation can be measured. T2K(-II) and NOvA experiments are overviewed and their inputs for this analysis are presented in section III. The outcomes of combined sensitivity between the T2K-II and NOvA experiments with constraint from reactor are presented in section $\mathbf{V}$.

\section{NEUTRINO OSCILLATION FRAMEWORK}

In three-flavor neutrino oscillation framework, the flavor definitive eigenstates are related to the mass definitive eigenstates by a $3 \times 3$ unitary PMNS matrix as shown in Eq. (1),

$$
\left(\begin{array}{c}
v_{e} \\
v_{\mu} \\
v_{\tau}
\end{array}\right)=U_{\mathrm{PMNS}}\left(\begin{array}{c}
v_{1} \\
v_{2} \\
v_{3}
\end{array}\right)=\left(\begin{array}{ccc}
U_{e 1} & U_{e 2} & U_{e 3} \\
U_{\mu 1} & U_{\mu 2} & U_{\mu 3} \\
U_{\tau 1} & U_{\tau 2} & U_{\tau 3}
\end{array}\right)\left(\begin{array}{c}
v_{1} \\
v_{2} \\
v_{3}
\end{array}\right) .
$$

\footnotetext{
${ }^{1}$ If neutrinos are Majorana particles, there are two phases added into the PMNS matrix. However the oscillation amplitudes are not sensitive to these two phases.
} 
The PMNS matrix can be parameterized by three mixing angles $\left(\theta_{12}, \theta_{13}, \theta_{23}\right)$ and a single Dirac phase $\delta_{C P}$, expressed in Eq. (2).

$$
U_{\mathrm{PMNS}}=\left(\begin{array}{ccc}
c_{12} c_{13} & s_{12} c_{13} & s_{13} e^{-i \delta_{C P}} \\
-s_{12} c_{23}-c_{12} s_{23} s_{13} e^{i \delta_{C P}} & c_{12} c_{23}-s_{12} s_{23} s_{13} e^{i \delta_{C P}} & s_{23} c_{13} \\
s_{12} s_{23}-c_{12} c_{23} s_{13} e^{i \delta_{C P}} & -c_{12} s_{23}-s_{12} c_{23} s_{13} e^{i \delta_{C P}} & c_{23} c_{13}
\end{array}\right),
$$

where $s_{i j}=\sin \theta_{i j}, c_{i j}=\cos \theta_{i j},\{i, j\}=\{1,2,3\}$ and the Dirac phase $\delta_{C P}$ represents the CP violation in lepton sector ${ }^{2}$. The probability for a $\alpha$-flavour neutrino with energy $E$ to change to $\beta$-flavour after travelling a distance of $L$ can be calculated as follows

$$
P\left(v_{\alpha} \rightarrow v_{\beta}\right)=\delta_{\alpha \beta}-4 \sum_{i>j} \mathfrak{R}\left[U_{\alpha i}^{*} U_{\beta i} U_{\alpha_{j}} U_{\beta_{j}}^{*}\right] \sin ^{2} \Delta_{i j}+2 \sum_{i>j} \mathfrak{I}\left[U_{\alpha i}^{*} U_{\beta i} U_{\alpha_{j}} U_{\beta_{j}}^{*}\right] \sin \left(2 \Delta_{i j}\right),
$$

where $\Delta_{i j}=\frac{\left(m_{i}^{2}-m_{j}^{2}\right) L}{4 E},\{i, j\}=\{1,2,3\}, i>j$. For antineutrinos, the oscillation probability can be obtained by replacing the mixing matrix elements with their complex conjugates. The $\mathrm{CP}$ violating phase $\delta_{C P}$ manifests itself in the difference between neutrino and antineutrino oscillations, as shown in Eq. (4).

$$
\begin{aligned}
\mathscr{A}_{\mathrm{CP}} & =P\left(v_{\alpha} \rightarrow v_{\beta}\right)-P\left(\bar{v}_{\alpha} \rightarrow \bar{v}_{\beta}\right)=4 \sum_{i>j} \mathfrak{I}\left[U_{\alpha i}^{*} U_{\beta i} U_{\alpha_{j}} U_{\beta_{j}}^{*}\right] \sin \left(2 \Delta_{i j}\right) \\
& = \pm 2 \delta_{\alpha \beta} \cos \theta_{13} \sin 2 \theta_{12} \sin 2 \theta_{23} \sin 2 \theta_{13} \sin \delta_{C P} \sin \Delta_{21} \sin \Delta_{32} \sin \Delta_{31},
\end{aligned}
$$

in which $\{\alpha, \beta\}=\{e, \mu, \tau\}$, the positive (negative) sign is applied based on (anti-)cyclic permutation of ordered flavor $(e, \mu, \tau)$. Apparently $\mathrm{CP}$ violation can be measured via the neutrino oscillation phenomenon if only three mixing angles are non-zero. The up-to-date neutrino data [8] shows that Nature supports this scenario and it opens the door to search CP violation in the lepton sector with neutrino oscillation measurements. This $\mathrm{CP}$ violation source might be a promising explanation for the matter-antimatter asymmetry in the Universe.

In practice, $\mathrm{CP}$ violation can be measured by comparing the rate of electron neutrinos appeared from muon neutrinos, $P\left(v_{\mu} \rightarrow v_{e}\right)$, with the one of electron antineutrinos appeared from muon anti-neutrinos, $P\left(\bar{v}_{\mu} \rightarrow \bar{v}_{e}\right)$ in the accelerator-based experiments or comparing the first with electron antineutrino disappearance in the reactor-based experiments. ${ }^{3}$

\footnotetext{
${ }^{2}$ If neutrinos are Majorana particles, the mixing matrix includes two additional phases which do not appear in the expression of oscillation probabilities.

${ }^{3}$ Accelerator-based measurements lead to an intrinsic $\delta_{C P}-\theta_{13}$ degeneracy while reactor-based measurements can precisely measure $\theta_{13}$. Their combined information thus can provide constraint on $\delta_{C P}$.
} 
The oscillation probability from muon neutrinos to electron neutrinos can be written to the first order approximation of matter effect [10] as follows

$$
\begin{aligned}
P\left(v_{\mu} \rightarrow v_{e}\right) \approx & 4 s_{13}^{2} s_{23}^{2} c_{13}^{2} \sin ^{2} \Delta_{31} \\
& -8 s_{13}^{2} s_{23}^{2} c_{13}^{2} \frac{a}{\Delta m_{31}^{2}}\left(2 s_{13}^{2}-1\right) \sin ^{2} \Delta_{31} \\
& +8 s_{13}^{2} s_{23}^{2} c_{13}^{2} \frac{a L}{4 E}\left(2 s_{13}^{2}-1\right) \sin \Delta_{31} \cos \Delta_{32} \\
& -8 s_{12} s_{13} s_{23} c_{12} c_{13}^{2} c_{23} \sin \delta_{C P} \sin \Delta_{21} \sin \Delta_{31} \sin \Delta_{32} \\
& +8 s_{12} s_{13} s_{23} c_{13}^{2}\left(c_{12} c_{23} \cos \delta_{C P}-s_{12} s_{13} s_{23}\right) \sin \Delta_{21} \sin \Delta_{31} \cos \Delta_{32} \\
& +4 s_{12}^{2} c_{13}^{2}\left(c_{12}^{2} c_{23}^{2}+s_{12}^{2} s_{13}^{2} s_{23}^{2}-2 s_{12} s_{13} s_{23} c_{12} c_{23} \cos \delta_{C P}\right) \sin ^{2} \Delta_{21},
\end{aligned}
$$

where $a=2 \sqrt{2} G_{F} n_{e} E=7.56 \times 10^{-5}\left[\mathrm{eV}^{2}\right]\left(\frac{\rho}{\mathrm{g} / \mathrm{cm}^{3}}\right)\left(\frac{E}{\mathrm{GeV}}\right), n_{e}$ is electron density of the matter and $\rho$ is density of the Earth. The appearance of $a$ in Eq. (5) is due to the matter effect which is rooted from the fact that electron neutrino passing through ordinary matter will interact weakly with electrons. For anti-neutrino counterpart, $P\left(\bar{v}_{\mu} \rightarrow \bar{v}_{e}\right)$ can be obtained from the Eq. (5) by replacing $\delta_{C P} \rightarrow-\delta_{C P}$ and $a \rightarrow-a$. In the Eq. (5), the first term dominates with current longbaseline neutrino experiments and is about 0.043 at the maximum of $\sin ^{2} \Delta_{31}$. The matter effect represented by constant $a$ involves to the second and third terms. While the term proportional to $\sin \delta_{C P}$ is called $C P$-violating since its contribution to total probability is opposite for neutrino and antineutrino. The fifth which contains $\cos \delta_{C P}$ is called $C P$-conserving term since its contribution is the same for neutrino and antineutrino. The last one depends on $\sin ^{2} \Delta_{21}$ and can be ignored in the case of long baseline experiments. At present landscape of neutrino oscillation, these channels are the only hope to provide information about $\delta_{C P}$. However challenges for these channel measurements are the smallness of oscillation amplitudes and their degeneracies with other oscillation parameters.

Along with the appearance channels, the accelerator-based long-baseline neutrino experiments typically can measure precisely the probabilities of $v_{\mu} \rightarrow v_{\mu}$ and $\bar{v}_{\mu} \rightarrow \bar{v}_{\mu}$, which can be expressed as [10]:

$$
\begin{aligned}
P\left(\bar{v}_{\mu} \rightarrow \vec{v}_{\mu}\right) \approx & 1+4 s_{23}^{2} c_{13}^{2}\left(s_{23}^{2} c_{13}^{2}-1\right) \sin ^{2} \Delta_{31} \\
& \pm 4 s_{23}^{2} c_{13}^{2} s_{13}^{2}\left(2 s_{23}^{2} c_{13}^{2}-1\right) \frac{2 a}{\Delta m_{31}^{2}} \sin ^{2} \Delta_{31} \\
& \pm 4 s_{23}^{2} c_{13}^{2} s_{13}^{2}\left(2 s_{23}^{2} c_{13}^{2}-1\right) \frac{a}{\Delta m_{31}^{2}} \Delta_{31} \sin 2 \Delta_{31} \\
& +4 s_{23}^{2} c_{13}^{2}\left(c_{12}^{2} c_{23}^{2}+s_{12}^{2} s_{13}^{2} s_{23}^{2}-2 s_{12} s_{13} s_{23} c_{12} c_{23} \cos \delta_{C P}\right) \Delta_{21} \sin 2 \Delta_{31},
\end{aligned}
$$

where positive (negative) signs are taken for neutrino (antineutrino) oscillations, respectively. Due to relative smallness of $\theta_{13}$, the first term is dominated in the accelerator-based long-baseline neutrino experiments. The measurement with this channel is essentially sensitive to mixing angle $\theta_{23}$ and $\Delta m_{31}^{2}$. In practice, neutrino oscillation analyses take advance of combining both appearance channel and disappearance channel in order to provide the most precise measurements of oscillation parameters and explore CP violation from constraint on $\delta_{C P}$. Fig. 1 (a) and Fig. 1 (b) show the oscillation probabilities of $\vec{v}_{\mu} \rightarrow \vec{v}_{e}$ as a function of neutrino energy at different true values 


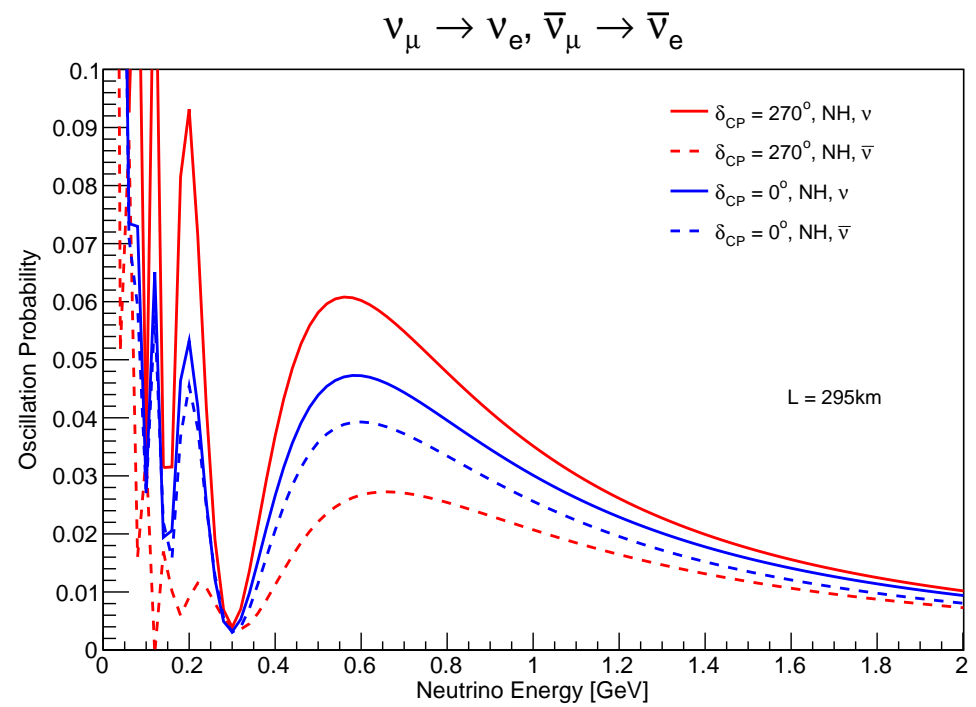

(a)

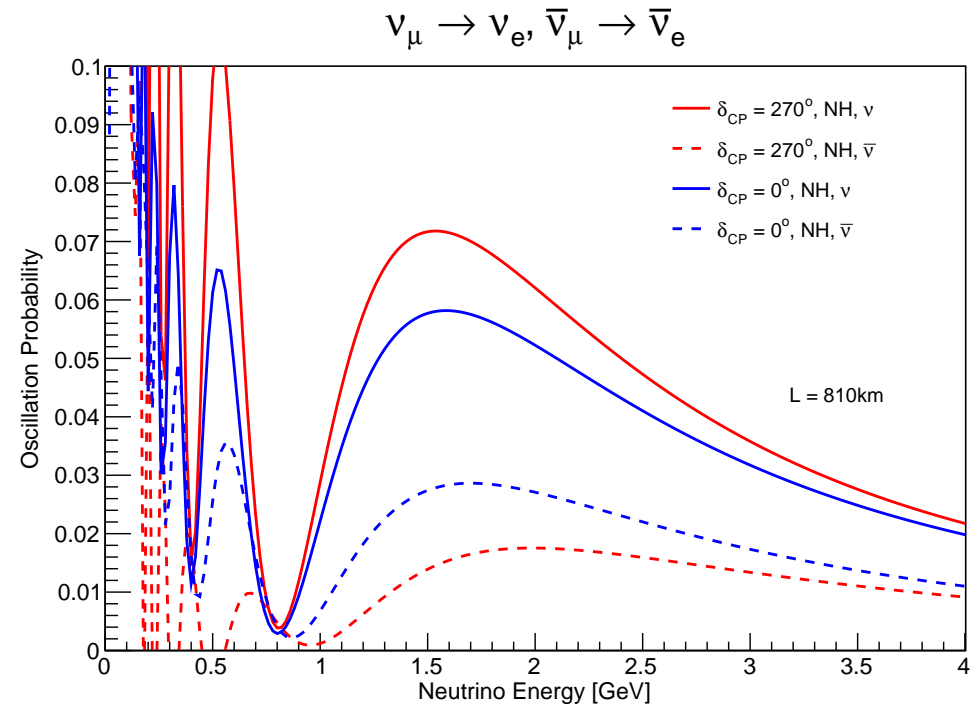

(b)

Fig. 1. Transition probabilities $P\left(v_{\mu} \rightarrow v_{e}\right)$ and $P\left(\bar{v}_{\mu} \rightarrow \bar{v}_{e}\right)$ as a function of neutrino energy for T2K baseline $L=295 \mathrm{~km}$ (a) and NOvA baseline $L=810 \mathrm{~km}$ (b).

of $\delta_{C P}$ for T2K baseline $L=295 \mathrm{~km}$ (with peak of neutrino flux at $0.6 \mathrm{GeV}$ ) and NOvA baseline $L=810 \mathrm{~km}$ (with peak of neutrino flux at $2 \mathrm{GeV}$ ), respectively. These two leading acceleratorbased long-baseline neutrino experiments will be discussed in detail in Section III. In the figures, the difference between solid and dashed blue lines indicates the matter effect, and the difference 


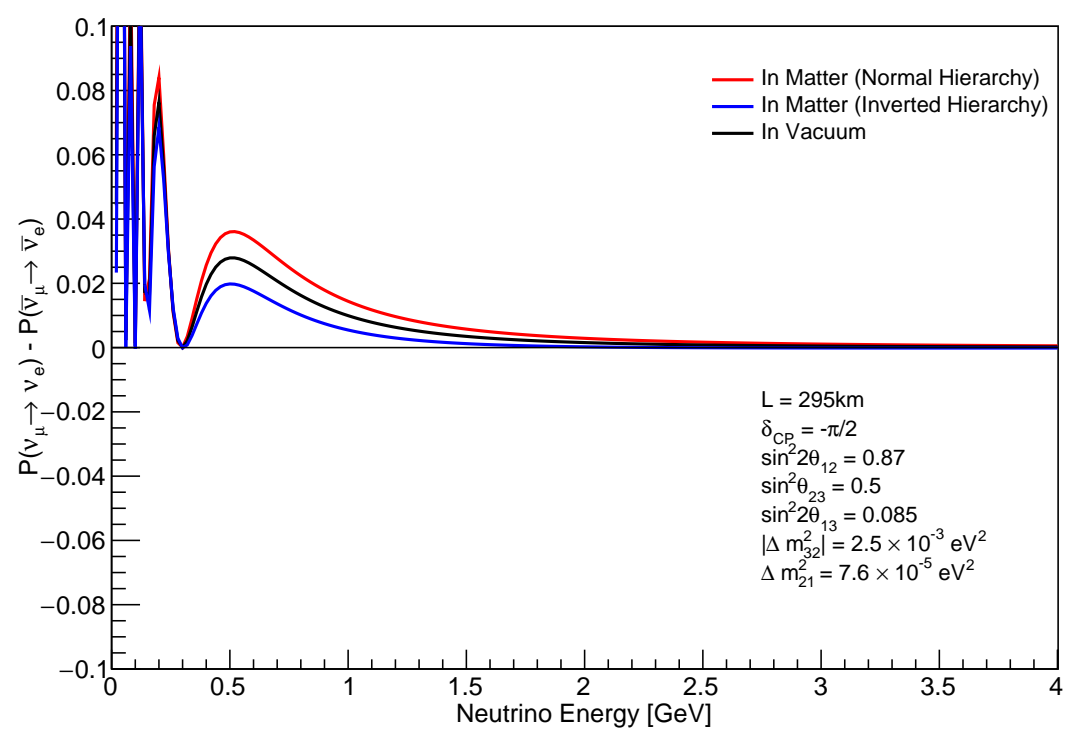

(a)

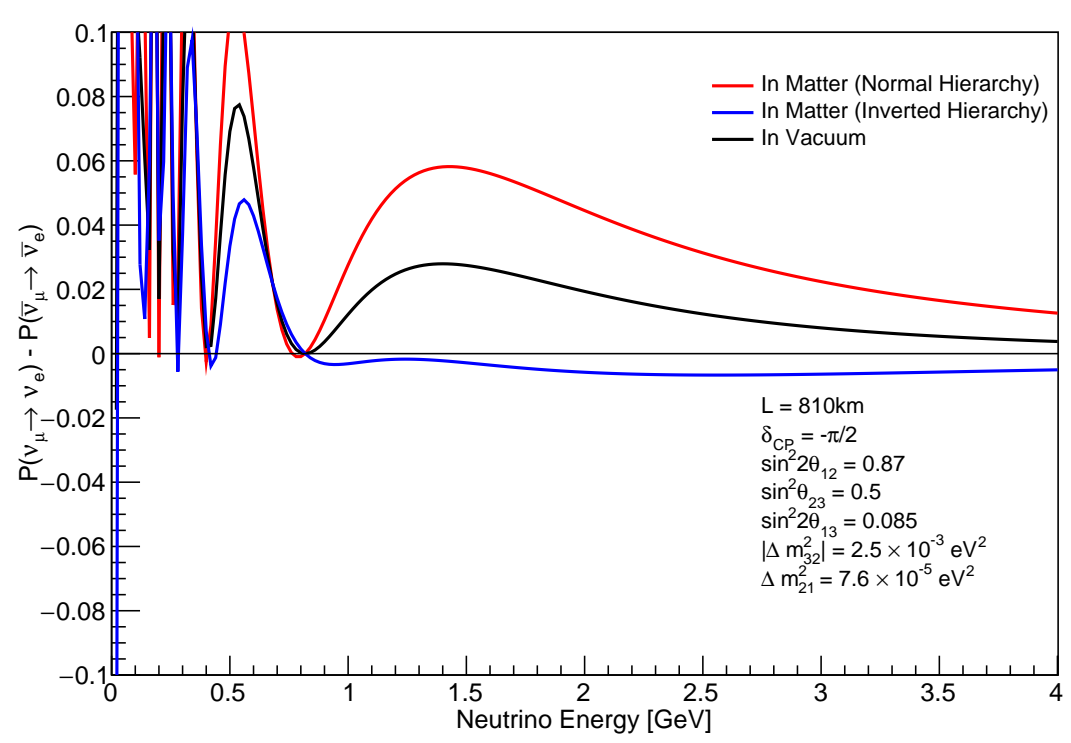

(b)

Fig. 2. Probability asymmetry $P\left(v_{\mu} \rightarrow v_{e}\right)-P\left(\bar{v}_{\mu} \rightarrow \bar{v}_{e}\right)$ as a function of neutrino energy for T2K baseline $L=295 \mathrm{~km}$ (a) and NOvA baseline $L=810 \mathrm{~km}$ (b).

between solid and dashed red lines shows the combined effect of both matter and CP violation. In the case of T2K experiment, the matter effect is much smaller than the CP violation effect. For NOvA the matter effect is larger due to its longer baseline. Fig. 2 (a) and Fig. 2 (b) illustrate the 
asymmetry of transition probabilities between neutrino mode and anti-neutrino mode, $\mathscr{A}_{\mathrm{CP}}$ shown in Eq. (4), in addition with the effect of matter as a function of energy for T2K and NOvA baselines, respectively. The plots are made with assumed values of oscillation parameters as listed in Table 1:

Table 1. Input values of oscillation parameters, taken from [11].

\begin{tabular}{|c|c|c|c|c|c|}
\hline & $\sin ^{2} 2 \theta_{12}$ & $\sin ^{2} 2 \theta_{13}$ & $\sin ^{2} \theta_{23}$ & $\Delta m_{21}^{2}$ & $\Delta m_{32}^{2}$ \\
\hline Value & 0.8704 & 0.085 & 0.5 & $7.6 \times 10^{-5} \mathrm{eV}^{2} / c^{4}$ & $2.5 \times 10^{-3} \mathrm{eV}^{2} / c^{4}$ \\
\hline
\end{tabular}

\section{T2K(-II) AND NOvA EXPERIMENTS}

At present, T2K and NOvA are two leading accelerator-based long baseline neutrino experiments in the world. We briefly describe these two experiments and inputs we use to study their combined sensitivity on CP violation search.

T2K (Tokai-to-Kamioka) [12] is an accelerator-based long-baseline neutrino oscillation experiment placed in Japan with three main complexes: (i) the J-PARC accelerator, (ii) the near detector suite placed at $280 \mathrm{~m}$ from the neutrino production target, and (iii) the far detector, SuperKamiokande, situated at $295 \mathrm{~km}$ away from the target. One of the most intense proton beam in the world, the J-PARC is used to produce a nearly pure $\vec{v}_{\mu}$ source. The near detector suite is designed to characterize the unoscillated neutrino beam while the far detector is used to observe the oscillation patterns. The primary goal of $\mathrm{T} 2 \mathrm{~K}$ is to observe oscillation from muon neutrinos to electron neutrinos, which has been achieved in 2013 [13]. With relatively large value of mixing angle $\theta_{13}$, the physics potential of $\mathrm{T} 2 \mathrm{~K}$ is revisited and $\mathrm{CP}$ violation search is placed as the central target. For the latest results [14], based on a total exposure of $2.23 \times 10^{21}$ POT which consists of $1.47 \times 10^{21}$ POT in $v$-mode and $0.76 \times 10^{21}$ POT in $\bar{v}$-mode, T2K firstly reports that $\mathrm{CP}$ conserving values $\left(0\right.$ and $\pi$ ) of $\delta_{C P}$ are out of the $2 \sigma$ C.L. range of its measurement for both normal and inverted mass hierarchies. By the year 2021 with a fully approved exposure of $7.8 \times 10^{21}$ POT, T2K will have sensitivity to the CP-violating phase $\delta_{C P}$ at $90 \%$ C.L. or higher over a significant range [9]. To intensively explore CP violation, T2K-II which is an extension of T2K operation up to 2026 is proposed to accumulate $20 \times 10^{21}$ POT [15]. This amount of data in combination with expected improvement in the neutrino beamline and neutrino oscillation analysis allows T2K to have $3 \sigma$ or higher significant sensitivity to CP violation. Also the oscillation parameters $\theta_{23}$ and $\Delta m_{31}^{2}$ can be measured at unprecedented levels.

In this paper, we use GLoBES software package [16] to study the physics potential of longbaseline neutrino experiments. For the inputs of T2K configuration in GLoBES, we follow closely the information in the paper [15]. The neutrino fluxes for both neutrino-mode and antineutrinomode operations are updated with the latest fluxes released by T2K collaboration. The efficiencies for detecting $v_{e}$ and $\bar{v}_{e}$ signals are set to be $66.3 \%$ and $69.7 \%$ respectively while the efficiencies for detecting $v_{\mu}$ and $\bar{v}_{\mu}$ signals are $72.6 \%$ and $80.2 \%$, respectively. The event rates for T2K far detector reconstructed from GLoBES with our T2K-II setup for the cases of true $\delta_{C P}=0,-\pi / 2,+\pi / 2$ are shown in the Table 2 for $\vec{v}_{e}$ appearance samples and Table 3 for $\vec{v}_{\mu}$ disappearance samples. The value here is consistent with [15] at acceptable level. 
Table 2. The $v_{e}$ and $\bar{v}_{e}$ event samples predicted to collect in T2K-II far detector for $20 \times 10^{21}$ POT with $50 \%$ effectively statistic improvement, sharing same amount for neutrino-mode and antineutrino-mode operations, at three different values of $\delta_{C P}=$ $-\pi / 2,0,+\pi / 2$. The event rates are consistent with result shown in [15].

\begin{tabular}{|c|c|c|c|c|c|c|c|}
\hline & $\delta_{C P}$ & Total & $\begin{array}{c}\text { Signal } \\
v_{\mu} \rightarrow v_{e}\end{array}$ & $\begin{array}{c}\text { Signal } \\
\bar{v}_{\mu} \rightarrow \bar{v}_{e}\end{array}$ & $\begin{array}{c}\text { Beam CC } \\
v_{e}+\bar{v}_{e}\end{array}$ & $\begin{array}{c}\text { Beam CC } \\
v_{\mu}+\bar{v}_{\mu}\end{array}$ & NC \\
\hline \multirow{2}{*}{$v$-mode $v_{e}$ sample } & $-\pi / 2$ & 558.8 & 448.6 & 2.8 & 73.3 & 1.8 & 32.3 \\
& 0 & 466.3 & 354.9 & 4.0 & 73.3 & 1.8 & 32.3 \\
& $+\pi / 2$ & 370.9 & 258.6 & 4.9 & 73.3 & 1.8 & 32.3 \\
\hline \multirow{2}{*}{$\bar{v}$-mode $\bar{v}_{e}$ sample } & $-\pi / 2$ & 115.8 & 19.8 & 52.3 & 29.2 & 0.4 & 14.1 \\
& 0 & 134.6 & 16.2 & 74.7 & 29.2 & 0.4 & 14.1 \\
& $+\pi / 2$ & 149.3 & 11.8 & 93.8 & 29.2 & 0.4 & 14.1 \\
\hline
\end{tabular}

Table 3. The $v_{\mu}$ and $\bar{v}_{\mu}$ event samples predicted to collect in T2K-II far detector for $20 \times$ $10^{21}$ POT with $50 \%$ effectively statistic improvement, sharing same amount of neutrinomode and antineutrino-mode operations at three values of $\delta_{C P}=-\pi / 2,0,+\pi / 2$. The event rates are consistent with result shown in [15].

\begin{tabular}{|c|c|c|c|c|c|c|c|}
\hline & $\delta_{C P}$ & Total & $\begin{array}{c}\text { Beam CC } \\
\nu_{\mu}\end{array}$ & $\begin{array}{c}\text { Beam CC } \\
\bar{v}_{\mu}\end{array}$ & $\begin{array}{c}\text { Beam CC } \\
v_{e}+\bar{v}_{e}\end{array}$ & $\begin{array}{c}v_{\mu} \rightarrow v_{e}+ \\
\bar{v}_{\mu} \rightarrow \bar{v}_{e}\end{array}$ & NC \\
\hline \multirow{2}{*}{$\nu$-mode } & $-\pi / 2$ & 2735.3 & 2393.3 & 158.2 & 1.6 & 7.2 & 175.0 \\
$v_{\mu}$ sample & 0 & 2737.0 & 2392.4 & 157.8 & 1.6 & 10.2 & 175.0 \\
& $+\pi / 2$ & 2740.7 & 2393.3 & 158.2 & 1.6 & 12.6 & 175.0 \\
\hline \multirow{2}{*}{$\bar{v}$-mode } & $-\pi / 2$ & 1283.5 & 507.8 & 707.9 & 0.6 & 1.0 & 66.2 \\
$\bar{v}_{\mu}$ sample & 0 & 1280.5 & 506.8 & 706.1 & 0.6 & 0.8 & 66.2 \\
& $+\pi / 2$ & 1283.1 & 507.8 & 707.9 & 0.6 & 0.6 & 66.2 \\
\hline
\end{tabular}

In addition, systematic uncertainties of all T2K-II signal samples are anticipated to go down to $4 \%$ compared with $5.5 \%-6.8 \%$ of current level. This can be achieved by reducing errors from neutrino fluxes, neutrino interaction models and detector model uncertainties.

NOvA (NuMI Off-axis $v_{e}$ Appearance) experiment [17] is an accelerator-based long-baseline neutrino experiment placed in United State. NOvA uses the intense and nearly pure $\vec{v}_{\mu}$ beam from NuMI (Neutrino at Main Injector), Fermilab and studies oscillations with two functionally identical detectors: near detector (0.3 kton) situated underground at Fermilab, Illinois and far detector (14 kton) installed on the surface in Ash River, Minesota, $810 \mathrm{~km}$ away from the neutrino production target. The detectors, are placed at an offset angle of $14 \mathrm{mrad}$ from the average neutrino beam line in order to achieve a narrow neutrino spectrum with peak at $2 \mathrm{GeV}$ and suppress the neutral current $\pi^{0}$ background. This configuration is optimized for observing $v_{e}$ signal. With 810 $\mathrm{km}$ baseline, the matter effect can change $v_{\mu} \rightarrow v_{e}$ appearance rate up to $\pm 30 \%$. In 2017, with an equivalent exposure of $6.05 \times 10^{20}$ POT, $33 v_{e}$ candidate was observed, clearly excess from $8.2 \pm 0.8$ background expected from MC. One of the major improvement in NOvA oscillation analysis is adopting machine learning algorithm, so-called Convolutional Visual Network [18] for the event-by-event classification of $v_{e}$ and $\bar{v}_{e}$. The gain from this new selection is equivalent to 
$30 \%$ effectively statistic increase. For the NOvA inputs, $61.0 \%$ and $71.5 \%$ signal efficiencies are used for selecting $v_{e}$ and $\bar{v}_{e}$ appearance samples, respectively. For the disappearance channels, the signal efficiencies for $v_{\mu}$ and $\bar{v}_{\mu}$ samples are $32.0 \%$ and $38.0 \%$, respectively. These numbers are based on the papers [19] and [20]. With an proposed operation up to the year 2024, NOvA is expected to accumulate a total exposure of $72 \times 10^{20}$ POT including $36 \times 10^{20}$ in $v$-mode operation and same amount for the $\bar{v}$-mode operation. The event rates for complete statistics of NOvA are shown in the Table 4. In the NOvA analysis, the background from cosmic ray in the considering signal samples is significant due to the fact that the far detector is on the Earth surface. Since it is not easy to implement the cosmic ray flux in GloBES, this background is manually added into the $v_{\mu}$ beam neutral-current (NC) channel. The systematic uncertainty by the end of NOvA operation is assumed to be $5 \%$ in GLoBES as it is provided.

Table 4. The $v_{e}$ and $\bar{v}_{e}$ event rates of full statistics of NOvA operation up to 2024 with $36 \times 10^{20}$ in $v$-mode and $36 \times 10^{20}$ POT in $\bar{v}$-mode. The rates are calculated at at three values of $\delta_{C P}=-\pi / 2,0,+\pi / 2$.

\begin{tabular}{|c|c|c|c|c|c|c|}
\hline & $\delta_{C P}$ & Total & Signal & $v_{\mu}$ beam CC & $v_{\mu}$ beam NC & $v_{e}$ beam \\
\hline & $-\pi / 2$ & 266.1 & 192.4 & 5.7 & 39.5 & 28.5 \\
$v$-mode & 0 & 239.4 & 165.7 & 5.7 & 39.5 & 28.5 \\
$v_{e}$ sample & $+\pi / 2$ & 195.6 & 121.9 & 5.7 & 39.5 & 28.5 \\
\hline & $-\pi / 2$ & 63 & 33.8 & 2.1 & 13 & 14.1 \\
$\bar{v}$-mode & 0 & 78.9 & 49.7 & 2.1 & 13 & 14.1 \\
$\bar{v}_{e}$ sample & $+\pi / 2$ & 87.5 & 58.3 & 2.1 & 13 & 14.1 \\
\hline
\end{tabular}

Table 5. The $v_{\mu}$ and $\bar{v}_{\mu}$ event rates of NOvA with $36 \times 10^{20}$ in $v$-mode and $36 \times$ $10^{20}$ POT in $\bar{v}-$ mode. The rates are calculated at at three values of $\delta_{C P}=-\pi / 2,0$, $+\pi / 2$.

\begin{tabular}{|c|c|c|c|c|}
\hline & $\delta_{C P}$ & Total & Signal & $v_{\mu}$ beam NC \\
\hline \multirow{3}{*}{$v$-mode $v_{\mu}$ sample } & $-\pi / 2$ & 546.3 & 512.5 & 33.8 \\
& 0 & 541.9 & 508.1 & 33.8 \\
& $+\pi / 2$ & 546.3 & 512.5 & 33.8 \\
\hline \multirow{2}{*}{$\bar{v}$-mode $\bar{v}_{\mu}$ sample } & $-\pi / 2$ & 276.9 & 271.3 & 5.6 \\
& 0 & 275 & 269.4 & 5.6 \\
& $+\pi / 2$ & 276.9 & 271.3 & 5.6 \\
\hline
\end{tabular}

\section{CONSTRAINT ON MIXING ANGLE $\theta_{13}$ FROM REACTOR-BASED EXPERIMENTS}

Essentially the measured $\mathrm{CP}$ phase with accelerator-based long-baseline neutrino experiments has a strong correlation with the mixing angle $\theta_{13}$ rooted from their relations in $P\left(\vec{v}_{\mu} \rightarrow \vec{v}_{e}\right)$ presented in the Eq. (5). To break this correlation and consequently increase the sensitivity in 
measuring the CP phase, constraint on $\theta_{13}$ measured via the $\bar{v}_{e}$ disappearance probability with the reactor-based experiments is used. The expression for this probability is shown in Eq. (7):

$$
P\left(\bar{v}_{e} \rightarrow \bar{v}_{e}\right)=1-\cos ^{4} \theta_{13} \sin ^{2} 2 \theta_{12} \sin ^{2} \Delta_{21}-\sin ^{2} 2 \theta_{13}\left(\cos ^{2} \theta_{12} \sin ^{2} \Delta_{31}+\sin ^{2} \theta_{12} \sin ^{2} \Delta_{32}\right) \text {. }
$$

Apparently there is no term of $\delta_{C P}$ in $P\left(\bar{v}_{e} \rightarrow \bar{v}_{e}\right)$ expression. At present, the precision on $\sin ^{2} 2 \theta_{13}$ is $6 \%$ [21] with best fit $\sin ^{2} 2 \theta_{13}=0.085$ [11]. Daya Bay reactor experiment has recently showed that they can achieve $3 \%$ precision on $\sin ^{2} 2 \theta_{13}$ by the year 2020 [22]. In this study we examine the $\delta_{C P}$ sensitivity with both two scenarios on the precision of $\theta_{13}$ by simply adjusting the statistics of reactor experiment in GloBES to achieve the defined precision. Fig. 3 shows the precision of $\theta_{13}$ for both scenarios we set up.

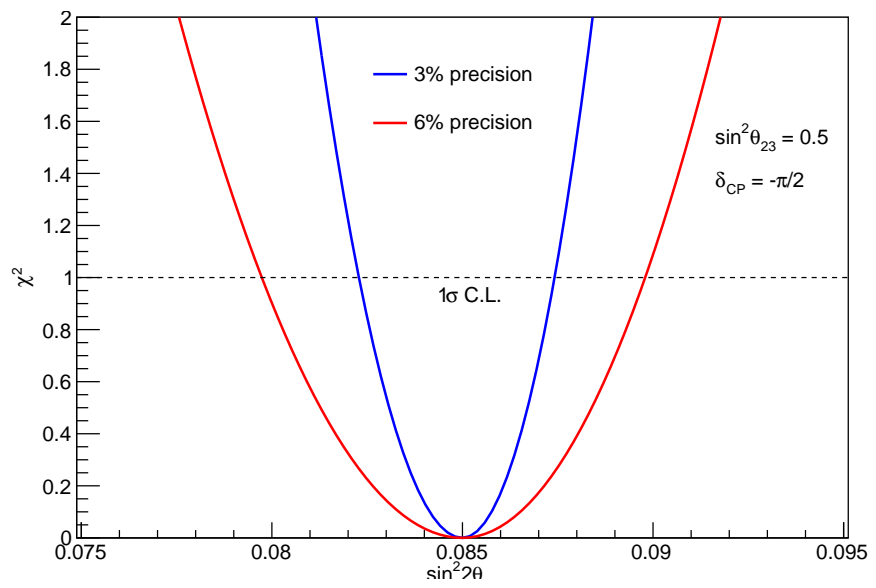

Fig. 3. Precision of $\theta_{13}$ from reactor-based experiments. The red line corresponds to $6 \%$ precision and the blue line corresponds to $3 \%$ precision of the current best fit value of $\sin ^{2} 2 \theta_{13}$.

\section{SENSITIVITY TO CP VIOLATION}

At present landscape, the value of $\delta_{C P}$ is known with marginal significant. Thus we explore the sensitivity of T2K-II and NOvA experiments on full range of this parameter. At each given value of $\delta_{C P}$, the minimal $\Delta \chi^{2}$ to exclude $\delta_{C P}=0$ and $\delta_{C P}= \pm 180^{\circ}$ are calculated. These values are then plotted as a function of true $\delta_{C P}$ in the meaning to exclude $\sin \delta_{C P}=0$. For all calculations shown below, we assume that the neutrino mass hierarchy would be known by the end of T2K-II and NOvA operations and to be normal. In the future we will explore for the case in which the neutrino mass hierarchy is unknown. Fig. 4(a) shows that T2K-II and NOvA respectively can achieve $3 \sigma$ C.L. and $2 \sigma$ C.L. to exclude CP-conserving values with current precision of mixing angle $\theta_{13}$ from reactor-based experiments, that agree with their expectations in [15] and [20]. Also the figure shows that the sensitivity to $\mathrm{CP}$ violation is increased when the ultimate constraint on $\theta_{13}$ from reactor-based experiments is taken into account. Particularly, when $\theta_{13}$ uncertainty reduces from $6 \%$ to $3 \%$, fractional region in which the $\mathrm{CP}$ conserving values $(0$ and $\pm \pi)$ can be excluded at $3 \sigma$ C.L. increases from $39.9 \%$ to $42.0 \%$ for T2K-II experiment. For NOvA, the $2 \sigma$ C.L.-excluded factional region increases from $40.8 \%$ to $41.4 \%$ when the uncertainty of $\theta_{13}$ is reduced. When the 
T2K-II and NOvA signal samples are combined and if the true value of $\delta_{C P}$ is close to $-\pi / 2$ as indicated by $\mathrm{T} 2 \mathrm{~K}$ result, the hypothesis of $\mathrm{CP}$ conservation in the lepton sector can be excluded at more than $4 \sigma$ with an fractional $\delta_{C P}$ region up to $32.4 \%$ as shown in Fig. $4($ b).

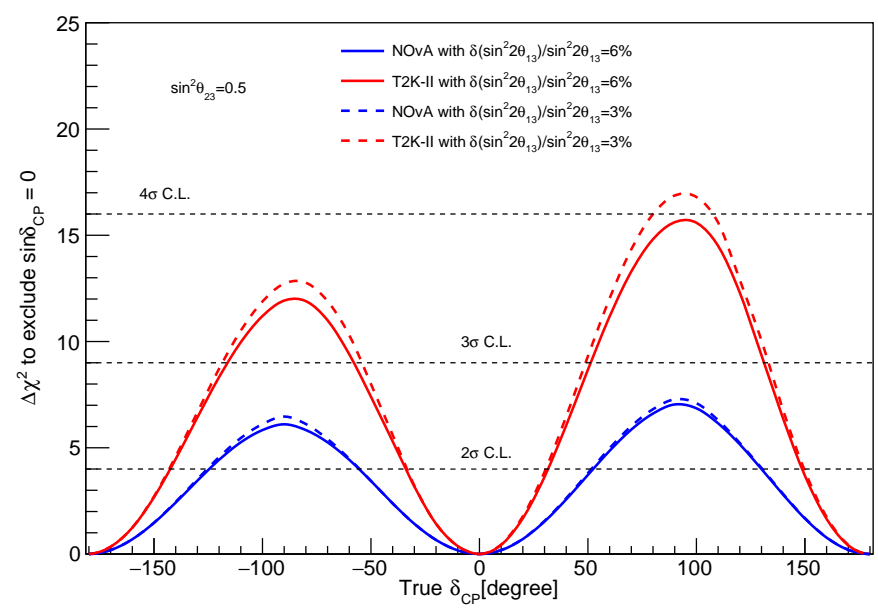

(a)

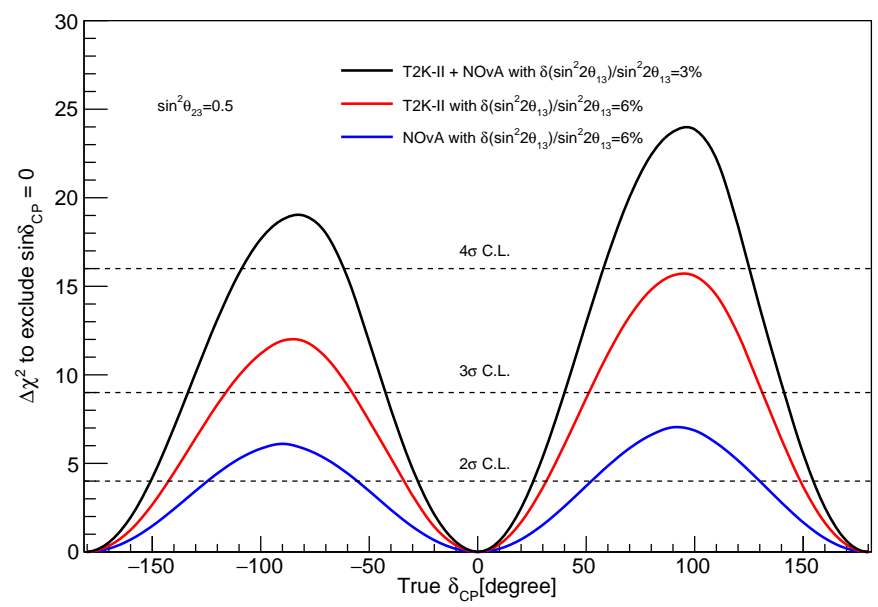

(b)

Fig. 4. (a) Sensitivity to CP violation as a function of true $\delta_{C P}$ for T2K-II and NOvA with $6 \%$ precision on $\sin ^{2} 2 \theta_{13}$ (solid red and blue lines, respectively), for T2K-II and NOvA with $3 \%$ precision on $\sin ^{2} 2 \theta_{13}$ (dashed red and blue lines, respectively).

(b) Sensitivity to $\mathrm{CP}$ violation as a function of true $\delta_{C P}$ for NOvA with $6 \%$ precision on $\sin ^{2} 2 \theta_{13}$ (solid blue line), T2K-II with $6 \%$ precision on $\sin ^{2} 2 \theta_{13}$ (solid red line) and $\mathrm{T} 2 \mathrm{~K}-\mathrm{II}+\mathrm{NOvA}$ with $3 \%$ precision on $\sin ^{2} 2 \theta_{13}$ (solid black line).

At the present, the sensitivity of $\mathrm{CP}$ violation in both $\mathrm{T} 2 \mathrm{~K}$ and NOvA experiments are limited predominantly by the statistics. However around 2024 to 2026 where we expect that these 
two experiments collect their full statistics, impact of systematics on the $\mathrm{CP}$ violation measurement should be considered seriously. In the above result, $4 \%$ and $5 \%$ uncertainties are assumed for the signal samples for T2K-II and NOvA respectively. We also check the scenario in which the uncertainties can be reduced to $2 \%$ level. The result is shown in Fig. 5. Evidently improving the systematics raises significantly the level of sensitivity to $\mathrm{CP}$ violation. We can make discovery of $\mathrm{CP}$ violation at $5 \sigma$ C.L. with fractional regions of $31.2 \%, 10.4 \%$ and $0 \%$ for $0.43,0.5$ and 0.6 of mixing angle $\sin ^{2} \theta_{23}$, respectively.

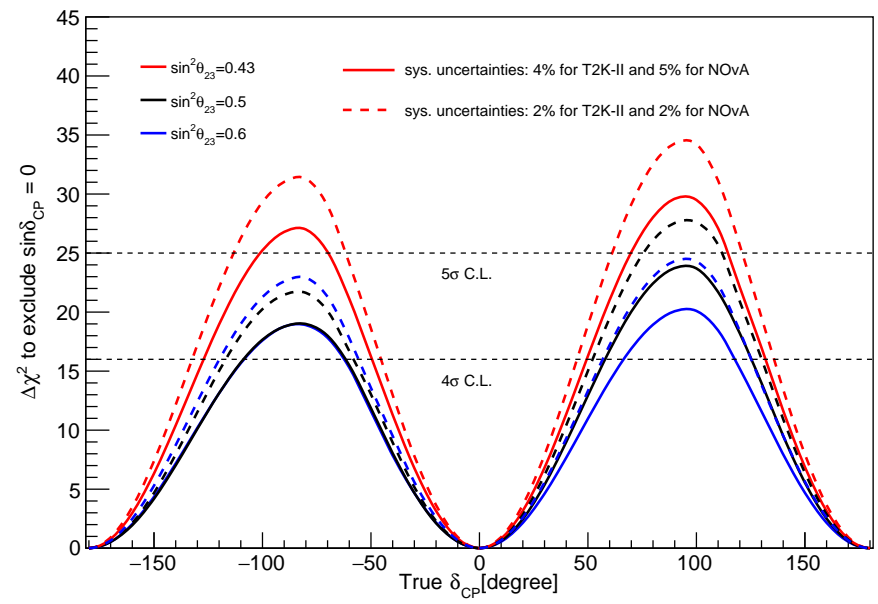

Fig. 5. Sensitivity to $C P$ violation as a function of true $\delta_{C P}$ with sys. uncertainties of $4 \%$ for $\mathrm{T} 2 \mathrm{~K}-\mathrm{II}+5 \%$ for NOvA + ultimate reactor constraint (solid lines), and $2 \%$ for T2K-II $+2 \%$ for NOvA + ultimate reactor constraint (dashed lines).

\section{CONCLUSIONS}

In this paper, we have studied the sensitivity to $\mathrm{CP}$ violation by combining the experiments T2K-II, which is proposed to run up to 2026, and NOvA, which can run up to 2024, with constraint from reactor-based experiments. With this combination, the $\mathrm{CP}$ conservation in lepton sector can be excluded at $4 \sigma$ C.L. significance if the true value of $\delta_{C P}$ is around $-\pi / 2$ as indicated by recent T2K measurement. The study also shows that precision measurement of mixing angle $\theta_{13}$ from the reactor-based experiments and improvements in the systematic uncertainties of measurement are crucial for the search of $\mathrm{CP}$ violation in the lepton sector. The $\mathrm{CP}$ violation can be discovered at $5 \sigma$ C.L. significance for a particular range of its value if $\theta_{13}$ precision reaches to $3 \%$ and the systematic uncertainty reduces to $2 \%$.

In the near future, we will consider an improvement of this study for more realistic description of both T2K-II and NOvA experiments by including efficiency as function of energy, smearing matrixes between true neutrino energy and reconstructed neutrino energy from experiments. Also adding measurements from atmospheric neutrino experiments such as Super-Kamiokande, IceCube, etc... will be considered to improve our sensitivity to $\mathrm{CP}$ violation measurements. 


\section{ACKNOWLEDGEMENT}

This research is funded by the National Foundation for Science and Technology Development (NAFOSTED) of Vietnam under contract No. 103.01-2017.76.

\section{REFERENCES}

[1] B. Pontecorvo, Sov. Phys. JETP 6 (1957) 429, [Zh. Eksp. Teor. Fiz.33,549(1957)].

[2] R. Davis, Jr., D. S. Harmer and K. C. Hoffman, Phys. Rev. Lett. 20 (1968) 1205.

[3] B. T. Cleveland, T. Daily, R. Davis, Jr., J. R. Distel, K. Lande, C. K. Lee, P. S. Wildenhain and J. Ullman, Astrophys. J. 496 (1998) 505.

[4] K. S. Hirata et al., Phys. Lett. B 205 (1988) 416, [,447(1988)].

[5] Z. Maki, M. Nakagawa and S. Sakata, Prog. Theor. Phys. 28 (1962) 870, [,34(1962)].

[6] Y. Fukuda et al., Phys. Rev. Lett. 81 (1998) 1562.

[7] Q. R. Ahmad et al., Phys. Rev. Lett. 87 (2001) 071301.

[8] C. Patrignani et al., Chin. Phys. C 40 (2016) 100001.

[9] K. Abe et al., PTEP 2015 (2015) 043C01.

[10] J. Arafune, M. Koike and J. Sato, Phys. Rev. D56 (1997) 3093, [Erratum: Phys. Rev.D60,119905(1999)].

[11] K. A. Olive et al., Chin. Phys. C 38 (2014) 090001.

[12] Y. Itow et al., The JHF-Kamioka neutrino project, Neutrino oscillations and their origin, 2001, pp. 239-248.

[13] K. Abe et al., Phys. Rev. Lett. 112 (2014) 061802.

[14] S. V. Cao, Latest results from T2K, Moriond EW 2018, La Thuile, Italy, March, 2018.

[15] K. Abe et al., (2016) .

[16] P. Huber, M. Lindner and W. Winter, Nucl. Phys. B 645 (2002) 3.

[17] D. S. Ayres et al., (2004) .

[18] A. Aurisano, A. Radovic, D. Rocco, A. Himmel, M. D. Messier, E. Niner, G. Pawloski, F. Psihas, A. Sousa and P. Vahle, JINST 11 (2016) P09001.

[19] M. A. Acero et al., (2018) .

[20] M. Sanchez, Nova results and prospects, June 2018.

[21] F. P. An et al., Phys. Rev. Lett. 115 (2015) 111802.

[22] J. Cao and K.-B. Luk, Nucl. Phys. B 908 (2016) 62. 